\title{
Effects on the Renin-Angiotensin System in Elderly Caregivers of Patients with Dementia: A Cross-Sectional Study
}

\author{
Akemi Hirano ${ }^{1, *}$, Yusuke Suzuki ${ }^{2}$, Toshio Hayashi ${ }^{3}$, Koichiro Ina ${ }^{4}$, Joji Onishi ${ }^{5}$ \\ ${ }^{1}$ Department of Adult Nursing, Shubun University, 6 Nikko-cho, Ichinomiya, Aichi 491-0938, Japan. \\ ${ }^{2}$ Centre for Community Liaison and Patient Consultations, Nagoya University Hospital, 65 Tsuruma, Showa, Nagoya, Aichi \\ 466-8550, Japan. \\ ${ }^{3}$ Department of Community and In-Home Nursing, Nagoya University Graduate School of Medicine, School of Health Sciences, \\ Daiko-Minami, Higashi-ku, Nagoya, Aichi 461-8673, Japan. \\ ${ }^{4}$ Department of Internal Medicine, Ina Clinic, 3-111 Hirabari, Tenpaku, Nagoya, Aichi 468-0011, Japan. \\ ${ }^{5}$ Department of Community Healthcare \& Geriatrics, Nagoya University Graduate School of Medicine, 65 Tsuruma, Showa, Nagoya, \\ Aichi 466-8550, Japan.
}

\begin{abstract}
How to cite this paper: Akemi Hirano, Yusuke Suzuki, Toshio Hayashi, Koichiro Ina, Joji Onishi. (2021) Effects on the Renin-Angiotensin System in Elderly Caregivers of Patients with Dementia: A Cross-Sectional Study. International Journal of Clinical and Experimental Medicine Research, 5(3), 243-248. DOI: 10.26855/ijcemr.2021.07.002
\end{abstract}

Received: March 22, 2021

Accepted: April 20, 2021

Published: May 10, 2021

*Corresponding author: Akemi Hirano, Department of Adult Nursing, Shubun University, 6 Nikko-cho, Ichinomiya, Aichi 491-0938, Japan.

Email: hirano@med.nagoya-u.ac.jp

\begin{abstract}
Background: Caregivers tend to have a lower estimated glomerular filtration rate (eGFR) than non-caregivers. Few studies have identified factors that affect the renin-angiotensin system in caregivers of dementia patients, who are under combined physical and psychological stress from caregiving. Purpose: The present study aimed to determine health factors that affect the renin-angiotensin system in caregivers of patients with dementia. Methods: A cross-sectional study was conducted among elderly caregivers of patients with dementia. Levels of renin and aldosterone were measured in each caregiver. Logistic regression analysis was performed. Results: Caregiver sex and BMI were identified as factors that influence the renin-angiotensin system in caregivers of dementia patients. Discussion: Chronic stress may increase aldosterone and renin levels in caregivers. Male caregivers had significantly higher renin levels than female caregivers, suggesting that the sympathetic activity in men may increase more markedly during caregiving than that observed in women in Japan. Factors that promote obesity, such as detrimental lifestyle habits that develop from the physical and mental fatigue of caregiving, may also have an impact. Conclusion: Increased support is needed for male caregivers, specifically for their caregiving efforts and health management. Other factors that increase caregiver BMI must also be identified.
\end{abstract}

\section{Keywords}

Caregiver Burden, Dementia, Renin, Aldosterone, Sex Differences, BMI

\section{Introduction}

The prevalence of hypertension is significantly higher in caregivers than in non-caregivers, and female caregivers have a lower estimated glomerular filtration rate (eGFR) than non-caregivers (adjusted OR: 6.54) [1]. Caregivers with hypertension exhibit a more marked decline in renal function compared to that observed in caregivers without hypertension. Similarly, caregivers with high diastolic blood pressure showed a more marked decline in renal function than that of caregivers with low diastolic blood pressure [2]. Factors that cause declines in caregiver 
renal function may include hypertension onset and exacerbation of chronic cardiovascular diseases.

Caregiving is a significant predictor of hypertension incidence [3], and for some elderly caregivers, their caregiving activities demand more than what their individual cardiovascular capacity may allow. Overburdened caregivers may develop health problems such as heart disease and may experience exacerbation of chronic disease [4]. One study noted increases in the progression of arrhythmia severity and frequency in elderly caregivers, finding that increases in blood pressure could be triggered by interruptions in sleep throughout the night due to the demand for nighttime care [5].

Stress evokes a reaction from the adrenal glands [6]. The endocrine system responds to stress by activating the hypothalamus-pituitary-adrenal (HPA) axis and the sympathetic adrenal medullary system, the latter of which influences the hypersecretion of adrenal hormones (in particular, glucocorticoids from the adrenal cortex and epinephrine from the adrenal medulla) [7], [8], [9].

Decreases in GFR and/or albuminuria are associated with a higher risk of death, particularly from cardiovascular causes; this risk extends to the elderly [10].

Adrenal glands play a central role in the body's response to stress, and proper adaptation of these glands is critical to maintaining homeostasis [11]. Cardiovascular disease has been found to be aggravated by stress in caregivers of dementia patients. However, few studies have identified factors that affect the renin-angiotensin system in caregivers of patients with dementia when the combination of physical and psychological stress of caregiving is present.

The present study aimed to identify health factors of caregivers of dementia patients that affect the renin-angiotensin system (RAS).

\section{Methods}

We conducted a cross-sectional study of elderly caregivers of patients with dementia. The biochemical data used in this study were as follows. Renin and aldosterone levels were measured in each of our study subjects.

Dependent variables were renin and aldosterone levels, analyzed by binomial logistic regression using the stepwise method. Any factors showing significant associations with these hormones were extracted as binary variables and categorized as below-median or above-median values for renin and aldosterone.

The study was approved by the Bioethics Review Committee of Nagoya University.

\section{Results}

The study subjects comprised $44.2 \%$ men. Renin and aldosterone levels in our study subjects were within the reference values. DBP was significantly higher in men (85.0) than in women (77.1) $(p=0.04) .48 .1 \%$ caregivers were taking $\mathrm{ARB}, \mathrm{ARB}+\mathrm{Ca}$ antagonists, alpha-beta blockers, ACE inhibitors, Ca antagonists, or platelet aggregation inhibitors to treat chronic diseases such as hypertension (Table 1).

Bivariate correlation matrix for hormone secretion and caregiver variables found a significant positive correlation between BMI and renin quantification ( $\mathrm{r}=0.456$, $\mathrm{p}=0.01$; Table 2 ). Significant correlations were also observed between renin quantification and sex $(r=-0.454, \mathrm{p}<0.01)$, number of ARB and other medications taken $(\mathrm{r}=0.327$, $\mathrm{p}<0.05)$, BMI ( $r=0.456, \mathrm{p}<0.001)$, and smoking history ( $\mathrm{r}=-0.317, \mathrm{p}<0.05)$ (Table 2$)$.

Factors showing significant associations with renin quantification included male sex (OR: 4.055; 95\% CI: 1.059-15.532), BMI (OR: 1.268; 95\% CI: 1.026-1.568) and the use of medications such as ARBs (OR: 4.037; 95\% CI: 1.182-13.782). No significant associations were observed between aldosterone and any of the independent variables examined (Table 3).

\section{Discussion}

The present study revealed that sex- and BMI-related health factors can influence the RAS in caregivers of patients with dementia.

Excess aldosterone and renin, which can stimulate the development of hypertension, promote vascular inflammation and fibrosis, remodeling, and endothelial dysfunction, all processes of which are associated with atherosclerosis [12], [13]. Increased sympathetic activity during stress increases renin production. The combination of living alone and chronic stress due to the presence of depressive symptoms led to significant increases in aldosterone $(p<0.01)$ and renin $(p=0.03)$ levels [14], suggesting that caregivers have higher aldosterone and renin levels due to chronic stress. 
Table 1. Characteristics of the study subjects

\begin{tabular}{|c|c|c|c|c|c|}
\hline \multirow[b]{2}{*}{ Caregiver } & \multicolumn{2}{|r|}{ Men } & \multicolumn{2}{|r|}{ Women } & \multirow[t]{2}{*}{$\mathrm{P}$} \\
\hline & Median & IQR (25-75\%) & Median & IQR (25-75\%) & \\
\hline Age & 77.0 & $75.0-79.0$ & 74 & $70.0-79.0$ & 0.219 \\
\hline Aldosterone (Ref: 35.7-240) (pg/mL) & 80.1 & $61.5-102.0$ & 96.9 & 70.8-121.5 & 0.095 \\
\hline Renin quantification (active) (Ref: $3.2 \sim 36$ ) (pg/mL) & 9.4 & 7.3-30.0 & 5.0 & 3.1-7.8 & 0.001 \\
\hline BMI & 23.7 & 21.3-25.9 & 22.9 & 19.6-24.9 & 0.217 \\
\hline SBP & 143.0 & $129.0-147.0$ & 139.0 & $120.0-148.5$ & 0.574 \\
\hline DBP & 85.0 & $76.0-91.0$ & 77.0 & $69.5-83.0$ & 0.040 \\
\hline Number of chronic diseases & 1.0 & $1.0-2.0$ & 1.0 & $1.0-2.0$ & 0.850 \\
\hline Taking any of medication (\%) & 48.1 & & & & \\
\hline \multicolumn{6}{|l|}{ Taking medications } \\
\hline ARB (\%) & 21.2 & & & & \\
\hline ARB + Ca antagonists (\%) & 1.9 & & & & \\
\hline alpha-beta blockers (\%) & 1.9 & & & & \\
\hline ACE inhibitors (\%) & 7.7 & & & & \\
\hline Ca antagonists (\%) & 26.9 & & & & \\
\hline platelet aggregation inhibitors (\%) & 17.3 & & & & \\
\hline
\end{tabular}

Notes: By Mann-Whitney Test, BMI: Body mass Index, SBP: systolic blood pressure, DBP: diastolic blood pressure, Taking any of medication: angiotensin receptor blockers (ARB), ARB + Ca antagonists, alpha-beta blockers, ACE inhibitors, Ca antagonists, or platelet aggregation inhibitors.

Table 2. Bivariate correlation matrix for hormone secretion and caregiver variables

\begin{tabular}{ccccccccc}
\hline & & Sex & age & Number of medications & SBP & BMI & smoking history \\
\hline Aldosterone & $\mathrm{r}$ & 0.233 & -0.178 & -0.104 & 0.006 & 0.198 & 0.008 \\
& $\mathrm{P}$ & 0.096 & 0.206 & 0.468 & 0.966 & 0.160 & 0.953 \\
Renin quantification (active) & $\mathrm{r}$ & $-0.454^{* *}$ & 0.086 & $0.327^{*}$ & 0.182 & $0.456^{* *}$ & $-0.317^{*}$ \\
& $\mathrm{P}$ & 0.001 & 0.545 & 0.019 & 0.197 & 0.001 & 0.022 \\
\hline
\end{tabular}

Notes: r: Spearman's correlation coefficient; P: level of significance, $\mathrm{p}:{ }^{*} \mathrm{p}<0.05,{ }^{* *} \mathrm{p}<0.01$; Number of medications: angiotensin receptor blockers (ARB), ARB + Ca antagonists, alpha-beta blockers, ACE inhibitors, Ca antagonists, or platelet aggregation inhibitors.

Table 3. Renin and aldosterone levels, analyzed by binomial logistic regression

\begin{tabular}{cccccccc}
\hline \multirow{2}{*}{ Dependent variable } & Variable (covariate) & B & $P$ & OR & Lower limit & Upper limit \\
\hline Aldosterone & No variables in the equation & & & & & \\
& Sex: Men & 1.400 & 0.041 & 4.055 & 1.059 & 15.532 \\
Renin quantification (active) & BMI & 0.238 & 0.028 & 1.268 & 1.026 & 1.568 & 1.610 \\
Renin quantification (active) & Number of taking medications & 0.237 & 0.051 & 1.268 & 0.999 & 1.194 & 14.247 \\
& Sex: Men & 1.417 & 0.025 & 4.125 & 1.182 & 13.782 \\
\hline
\end{tabular}

Notes: Each variable was entered into the model as a dichotomous variable indicating whether the participant was within or outside the median values (renin, 7.45; aldosterone, 89.2). In the logistic regression analysis, dependent variables were dichotomized as high (1) or low (0) relative to the median values (aldosterone $<89.2=0$; $>89.2=1$; renin $<7.45=0 ;>7.45=1$ ); B: partial regression coefficient, P: level of significance, $95 \% \mathrm{CI}$ : $95 \%$ confidence interval, BMI: Body Mass Index. Presence of taking medication: The use of medications (angiotensin receptor blockers (ARB), ARB + Ca antagonists, alpha-beta blockers, ACE inhibitors, Ca antagonists, or platelet aggregation inhibitors).

Sex, BMI, smoking history, and number of ARBs and other medications taken to treat chronic disease were found to be significantly associated with renin levels in our study subjects. Enhanced sympathetic activity increases 
the production of renin and angiotensin 2 [15] [16]. The higher renin levels observed in our male caregivers suggest that in general, their nervous systems may respond more markedly to the demands of caregiving than do those of female caregivers. One possible explanation for this may be the unfamiliarity among male caregivers toward caregiving, relative to that of female caregivers in Japan.

Forty-eight percent of our study subjects were taking ARB, ARB+Ca antagonists, $\alpha \beta$-blockers, ACE inhibitors, $\mathrm{Ca}$ antagonists, or platelet aggregation inhibitors. Median systolic blood pressure was $143 \mathrm{mmHg}$ in men, which is somewhat high, but we assume that it needed to be controlled. Subjects were not overly obese. A few of our study subjects were smokers, and higher renin levels were found to correlate negatively with smoking. The superimposition of a disease process, or the injudicious prescription of a drug, inhibiting renin release or angiotensin II production [17]. In other words, the RAS may be impaired in subjects taking multiple medications for blood pressure control.

Indeed, increased expression of some components of the RAS has been found to be significantly associated with cardiovascular disease and obesity [18]. Gene expression of RAS components is reportedly higher in the visceral adipose tissue of obese humans [19], suggesting that renin may be elevated in obese individuals. In addition, factors that promote obesity may be influenced by deteriorating lifestyle habits that develop as a result of the physical and mental fatigue of caregiving.

Chronic smoking increases plasma renin activity, which leads to higher aldosterone production, presumably via enhanced generation of angiotensin II. This may partly explain the greater vasoconstrictive reactivity typical of the arteries of chronic smokers [20]. In the present study, renin levels were negatively correlated with smoking, suggesting that factors other than smoking are likely involved.

Factors that influence renin levels included male sex, caregiver BMI, and use of medications such as ARB. Diastolic blood pressure was higher in men than in women. In men, testosterone amplifies the pressor pathways of the RAS [21]. Our findings were consistent with previous studies that found increased RAS activity to be associated with obesity and cardiovascular disease [22]. Renin may be more susceptible to drug responses such as ARB. Renin levels are known to decrease with age [23], and those with higher renin levels seem to have more brain and cardiovascular events [24] [25] [26]. This suggests that high renin levels may contribute to the development of hypertension, and therefore may represent a cardiovascular risk factor.

None of the variables we examined was found to correlate significantly with aldosterone level. Plasma aldosterone levels are reportedly high in patients with metabolic syndrome, suggesting that aldosterone may contribute to the increased risk of cardiovascular disease [27]. Visceral fat may secrete substances that promote aldosterone production. The lack of aldosterone elevation in the present study subjects may have been due to the fact that BMI among our subjects was not so indicative of obesity.

\section{Conclusions}

This study was able to identify factors that influence the secretion of renin and aldosterone, hormones of the RAS, in caregivers of patients with dementia. We identified caregiver sex (male) and BMI as significant variables that influenced caregiver renin levels. One limitation of this study is that we did not increase the sample size and investigate factors related to lifestyle. Future studies should investigate how best to support the health and caregiving efforts of male caregivers and identify factors that increase caregiver BMI.

\section{Acknowledgements}

We thank the participants for their cooperation in this study.

\section{References}

[1] Torimoto-Sasai, Y., Igarashi, A., Wada, T., Ogata, Y., Yamamoto-Mitani, N. (2015). Female family caregivers face a higher risk of hypertension and lowered estimated glomerular filtration rates: a cross-sectional, comparative study. BMC Public Health, 22(15): 177. doi: 10.1186/s12889-015-1519-6. PMID: 25927998; PMCID: PMC4340290.

[2] von Kanel, R., Mausbach, B. T., Dimsdale, J. E., Mills, P. J., Patterson, T. L., Ancoli-Israel, S., Ziegler, M. G., Roepke, S. K., Chattillion, E. A., Allison, M., Grant, I. (2012). Effect of chronic dementia caregiving and major transitions in the caregiving situation on kidney function: a longitudinal study. Psychosom Med., 74(2): 214-220. doi:10.1097/PSY.0b013e3182408c14. Epub 2012 Jan 27. PMID: 22286846; PMCID: PMC3384744.

[3] Capistrant, B. D., Moon, J. R., Glymour, M. M. (2012). Spousal caregiving and incident hypertension. Am J Hypertens, 25(4): 437-443. doi: 10.1038/ajh.2011.232. Epub 2011 Dec 22. PMID: 22189941; PMCID: PMC3836043. 
[4] Nishimura, Y. (1998). Cardiovascular response relating to stress on the elderly caregivers (part1)-fosuced on caregiving act of women caregivers. J Jpn Acad Nurs Sci., 18(3): 87-95. doi: 10.5630/jans1981.18.3_87.

[5] Nishimura, Y. (1999). Cardiovascular response relating to stress on the elderly caregivers (part2)-fosuced on night caregiving. J Jpn Acad Nurs Sci., 19(1): 13-22. doi: 10.5630/jans1981.19.1_13.

[6] Rubin de Celis, M. F., Bornstein, S. R., Androutsellis-Theotokis, A., Andoniadou, C. L., Licinio J, Wong ML, Ehrhart-Bornstein M. (2016). The effects of stress on brain and adrenal stem cells. Mol Psychiatry, 21(5): 590-593. doi: 10.1038/mp.2015.230. Epub 2016 Jan 26. Erratum in: Mol Psychiatry. 2016 May; 21(5): 722. de Celis, M F R [Corrected to Rubin de Celis, M F]. PMID: 26809844.

[7] Axelrod, J. and T. D. Reisine. (1984). Stress hormones: their interaction and regulation. Science, 224: $452-459$.

[8] Chrousos, G. P. and P. W. Gold. (1992). The concepts of stress and stress system disorders. Overview of physical and behavioral homeostasis. JAMA, 267: 1244-1252.

[9] Pervanidou, P. and G. P. Chrousos. (2007). Posttraumatic stress disorder in children and adolescents : from Sigmund Freud's "trauma” to psychopathology and the (dys) metabolic syndrome. Horm Metab Res., 39: 413-419.

[10] Dukkipati, R., Adler, S., Mehrotra, R. (2008). Cardiovascular implications of chronic kidney disease in older adults. Drugs Aging, 25(3): 241-53. doi: 10.2165/00002512-200825030-00006. PMID: 18331075.

[11] Rubin de Celis, M. F., Garcia-Martin, R., Wittig, D., Valencia, G. D., Enikolopov, G., Funk, R. H., et al. (2015). Multipotent glia-like stem cells mediate stress adaptation. Stem Cells, 33: 2037-2051.

[12] Schiffrin, E. L. (2006). Effects of aldosterone on the vasculature. Hypertension, 47(3): 312-318. doi: 10.1161/01.HYP.0000201443.63240.a7. Epub Jan 23. PMID: 16432039.

[13] Benard, L., Milliez, P., Ambroisine, M. L., Messaoudi, S., Samuel, J. L., Delcayre, C. (2009). Effects of aldosterone on coronary function. Pharmacol Rep., 61(1): 58-66. doi: 10.1016/s1734-1140(09)70007-6. PMID: 19307693.

[14] Häfner, S., Baumert, J., Emeny, R. T., Lacruz, M. E., Bidlingmaier, M., Reincke, M., Kuenzel, H., Holle, R., Rupprecht, R., Ladwig, K. H.; MONICA/KORA Study Investigators. (2012). To live alone and to be depressed, an alarming combination for the renin-angiotensin-aldosterone-system. Psychoneuroendocrinology, 37(2): 230-237. doi: 10.1016/j.psyneuen.2011.06.007. Epub 2011 Jul 13. PMID: 21742440.

[15] Yang, G., Xi, Z. X., Wan, Y., Wang, H., Bi, G. (1993). Changes in circulating and tissue angiotensin II during acute and chronic stress. Biol Signals, 2(3): 166-172. doi: 10.1159/000109488. PMID: 8004155.

[16] Yang, G., Wan, Y., Zhu, Y. (1996). Angiotensin II-an important stress hormone. Biol Signals, 5(1): 1-8. doi: 10.1159/000109168. PMID: 8739318.

[17] Bauer, J. H. (1993). Age-related changes in the renin-aldosterone system. Physiological effects and clinical implications. Drugs Aging, 3(3): 238-245. doi: 10.2165/00002512-199303030-00005. PMID: 8324299.

[18] Thethi, T., Kamiyama, M., Kobori, H. (2012). The link between the renin-angiotensin-aldosterone system and renal injury in obesity and the metabolic syndrome. Curr Hypertens Rep., 14(2): 160-169.

[19] Pinheiro, T. A., Barcala-Jorge, A. S., Andrade, J. M. O., Pinheiro, T. A., Ferreira, E. C. N., Crespo, T. S., et al. (2017). Obesity and malnutrition similarly alter the renin-angiotensin system and inflammation in mice and human adipose. J Nutr Biochem, 48: 74-82.

[20] Laustiola, K. E., Lassila, R., Nurmi, A. K. (1988). Enhanced activation of the renin-angiotensin-aldosterone system in chronic cigarette smokers: a study of monozygotic twin pairs discordant for smoking. Clin Pharmacol Ther., 44(4): 426-430. doi: 10.1038/clpt..175. PMID: 3048842.

[21] Reckelhoff, J. F. (2008). Sex and sex steroids in cardiovascular-renal physiology and pathophysiology. Gend Med., 5 Suppl A: S1-2. doi: 10.1016/j.genm.2008.03.001. PMID: 18395674.

[22] Thethi, T., Kamiyama, M., Kobori, H. (2012). The link between the renin-angiotensin-aldosterone system and renal injury in obesity and the metabolic syndrome. Curr Hypertens Rep., 14(2): 160-169. doi: 10.1007/s11906-012-0245-z. PMID: 22302531; PMCID: PMC3337881.

[23] Hayduk, K., Krause, D. K., Kaufmann, W., Huenges, R., Schillmöller, U., Umbehaun, V. (1973). Age-dependent changes of plasma renin concentration in humans. Clin Sci Mol Med Suppl., 45 Suppl 1: 273s-8. doi: 10.1042/cs045273s. PMID: 4522174.

[24] Brunner, H. R., Laragh, J. H., Baer, L., Newton, M. A., Goodwin, F. T., Krakoff, L. R., Bard, R. H., Bühler, F. R. (1972). Essential hypertension: renin and aldosterone, heart attack and stroke. N Engl J Med., 286(9): 441-449. doi: 10.1056/NEJM197203022860901. PMID: 4257928.

[25] Park, B. E., Yang, D. H., Kim, H. J., Park, Y. J., Kim, H. N., Jang, S. Y., Bae, M. H., Lee, J. H., Park, H. S., Cho, Y., Chae, S. C. (2020). Incremental Predictive Value of Plasma Renin Activity as a Prognostic Biomarker in Patients with Heart Failure. J Korean Med Sci., 2020 Nov 2; 35(42): e351. doi: 10.3346/jkms.2020.35.e351. PMID: 33140588; PMCID: PMC7606887.

[26] Jang, S. Y., Chae, S. C., Bae, M. H., Lee, J. H., Yang, D. H., Park, H. S., Cho, Y., Cho, H. J., Lee, H. Y., Oh, B. H., Choi, J. O., Jeon, E. S., Kim, M. S., Lee, S. E., Kim, J. J., Hwang, K. K., Cho, M. C., Baek, S. H., Kang, S. M., Choi, D. J., Yoo, B. S., Ahn, 
Y., Kim, K. H., Park, H. Y. (2018). Effect of renin-angiotensin system blockade in patients with severe renal insufficiency and heart failure. Int J Cardiol., 2018 Sep 1; 266: 180-186. doi: 10.1016/j.ijcard.2018.03.016. PMID: 29887444.

[27] Bochud, M., Nussberger, J., Bovet, P., Maillard, M. R., Elston, R. C., Paccaud, F., Shamlaye, C., Burnier, M. (2006). Plasma aldosterone is independently associated with the metabolic syndrome. Hypertension, 48(2): 239-245. doi: 10.1161/01.HYP.0000231338.41548.fc. Epub 2006 Jun 19. PMID: 16785327. 